\title{
Experimental tests on ultrasonic flow meter performance in full-flowing pipes
}

\author{
G. Ciaravino \& L. Ciaravino \\ Department of Hydraulic, Geotechnical and Environmental Engineering, \\ University of Naples Federico II, Italy
}

\begin{abstract}
Tests based on non destructive techniques are fundamental for measurements on fully operational plants. This work details the results obtained in experimental tests on full-flowing pipes made of a variety of materials highlighting some of the characteristics and limitations in the performance of the ultrasonic flow meter which must be taken into account. The research carried out using a Doppler ultrasonic flow meter (UDFM) illustrated anomalies in the device's function on steel pipes that have been in service for many years and in which significant internal irregularities and incrustations were present. With the internal surface of the pipe wall in such conditions, the acoustic signal loses its coherence, in passing through the pipe wall twice, thus making its detection difficult for the receiver. Further tests conducted on acrylic pipes, characterized by an extremely smooth internal surface and under the same experimental conditions, did not present the running anomalies encountered in the old steel pipes.
\end{abstract}

Keywords: ultrasonic flow meter, full-flowing pipe, performance experiment, accuracy tests.

\section{Introduction}

Measuring quantities such as velocity and flow rate of fluid transport networks or systems is necessary both to verify their correct function and for economical and managerial evaluations. If appropriate measuring devices are not positioned in advance at appropriate sections, this operation can be complicated to carry out under service conditions and involve disturbing or even interrupting the operation of the network or system. Consequently, developing devices that permit testing with essentially non intrusive and therefore non-destructive (NDT) 
methods seems attractive in order to permit fundamental measurements in any section and without interrupting or disturbing ongoing operations.

Among the measuring devices that have these characteristics there are the ultrasonic flow meters (UFM) [1]. The UFM is a non-intrusive device devoid of moving mechanical parts (so it does not cause any head loss) that has the ability to measure velocity in a bi-directional way, independently from the size of the pipe and without generally requiring calibration [2-5]. These characteristics make the UFM particularly useful for flow rate measurements (even continuously) on pipes both of wide and small diameter, tests in hydraulic machinery, hydroelectric power plants and in laboratory installations in which the flow must under no circumstances be disturbed [6-13]. In several works [1419] it has been shown how UFMs are also valuably used in measuring the velocity and therefore the flow rate in sewers, open channels and rivers.

In this article the results obtained in specific experimental tests conducted on pipes under pressure are reported showing some attributes and limitations in the performance of UFMs that should be considered.

\section{Principles of ultrasonic velocimetry}

Currently various types of ultrasonic flow meters are available for measurements in full-flowing pipes. To summarize two types can be considered: the Doppler ultrasonic flow meter (UDFM or UDV = ultrasonic Doppler velocimeter), the first to be built, and the Transit-time ultrasonic flow meter (UTFM or UTV = ultrasonic Transit-time velocimeter). In the event of measurements on functioning plants, both systems can use clamp-on transducers (transmitter and receiver) mounted to the outside of the pipe wall.

In the UDFM a transmitting transducer produces an ultrasonic beam (between 0.5 and $1 \mathrm{MHz}$ ) that passes through the pipe walls, penetrates the liquid, hits any particles of different density moving with the fluid, and is then reflected with a different frequency. The acoustic signals reflected by the particles are captured by a receiving transducer that analyses the frequency. The movement of the reflecting particles actually determines a Doppler effect that produces frequency shifts of the ultrasonic beam emitted. The mean value of the frequency shifts can be directly correlated to the mean velocity of the particles moving with the fluid. In truth because of the presence of many particles, moving at different speeds, the acoustic signals captured are of various frequencies, whose mean shift value can be correlated to the average velocity of the reflecting particles and thus, of the fluid transporting them. Therefore, save for any experimental correction coefficients, it is possible to measure the flow rate. The use of the UDFM requires the presence of sonically reflective materials such as solid particles (or entrained air bubbles) in the liquid flowing through the pipe: indeed the performance of the flow meter is tied to the particle density and particle concentration and distribution in the measurement section, as well as to the temperature and the sonic conductivity of the liquid. The use of an UDFM, therefore, is based on the fundamental hypothesis that the particles are moving at 
the same velocity as the fluid [20-22]. The principles of acoustic measurement with Doppler flow meters are mathematically expressed by the eqn. (1):

$$
V_{F}=\frac{V_{S} \cdot f_{D}}{2 \cdot \sin \theta \cdot f_{T}},
$$

where $V_{F}$ is the flow velocity, $V_{S}$ the sonic velocity of the fluid, $f_{D}$ is the Doppler frequency shift produced by particle, $f_{T}$ the transmitter frequency, $\theta$ the angle of entry of $f_{T}$ in the flow. Moreover according to Snell's law:

$$
\frac{V_{S}}{\sin \theta}=\frac{V_{T}}{\sin \theta_{T}},
$$

where $V_{T}$ is the sonic velocity of transmitter material and $\theta_{T}$ the angle of transmitter beam; consequently:

$$
V_{F}=\frac{V_{T} \cdot f_{D}}{2 \cdot \sin \theta_{T} \cdot f_{T}} .
$$

The UDFM measures the frequency shift produced from a large number of particles providing a frequency spectrum. Using microcomputer technology, an average value of velocity and the pipe flow rate is derived from the spectrum.

The UTFM is based on the principle that the transit-time of an acoustic signal is different in the upstream and in downstream directions: the difference can once again be measured using microcomputer technology and can be related to the flow velocity $[14,16,23]$.

In particular, in the downstream direction the flowing water velocity $V_{P d}$ along the acoustic path is:

$$
V_{P d}=\frac{L_{P}}{t_{d}}-V_{S},
$$

where $L_{P}$ is acoustic path length between the faces of the transducers, $t_{d}$ the acoustical signal downstream travel time and again $V_{S}$ is the sonic velocity of fluid. In the upstream direction the flowing water velocity $V_{P u}$ along the acoustic path is:

$$
V_{P u}=V_{S}-\frac{L_{P}}{t_{u}},
$$

where $t_{u}$ is the acoustic signal's upstream travelling time. When eqns. (3) and (4) are added the average path velocity $V_{P}$ is mathematically expressed by eqn. (6): 


$$
V_{P}=\frac{L_{P}}{2}\left(\frac{1}{t_{d}}-\frac{1}{t_{u}}\right)
$$

with the $\beta$ angle between the axis of the pipe and the straight line (acoustic path) connecting the faces of the transducers, eqn. (6) defines the average velocity of the fluid $V_{F}$ :

$$
V_{F}=\frac{L_{P}}{2 \cdot \cos \beta}\left(\frac{1}{t_{d}}-\frac{1}{t_{u}}\right) .
$$

Eqns. (4), (5), (6) and (7) are based on the hypothesis that velocity $V_{S}$ varies with the conductance and temperature of water. Moreover entrained gases and/or suspended particles affect the acoustic signal strength in the UTFM. Consequently the UTFM is generally used in clean stream-flow, where the ultrasonic beam is not attenuated or interrupted by particles moving with the fluid.

\section{Experimental tests}

In the context of developing an experimental installation designed to study cavitation in pumps, measuring the flow rate with an utrasonic flow meter was proposed. In fact, in order to achieve even very intense cavitation conditions, it was predicted that depression values determined within the installation would have made the use of devices such as diaphragms and Venturi meters practically impossible. Furthermore there was also the prediction that the current could drag cavitation bubbles and so, in order not to disturb such delicate hydrodynamics any way, it was necessary to adopt a non intrusive measuring device. For this reason and because the fluid feeding the experimental installation (from the closed circuit supply network of the Department of Hydraulic of the University of Naples) contains a considerable number of suspended particles, an ultrasonic Doppler flow meter was chosen. In truth the presence of a noteworthy quantity of particles in the supply circuit has been documented (and used) many times when, during experimental work in which surveys of velocity profiles were carried out on fluid currents using a Laser Doppler Velocimeter, no problems of signal interruption of the device (drop out phenomenon) were found that would be due to a scarcity of suspended particles. Consequently in order to verify the reliability of the device under the previously described experimental conditions, a number of tests were carried out conferring more general validity to the results.

The first test series (Series I) was carried out mounting the UDFM exteriorly to one of the pipes (accurately following the prescribed procedure) that is part of the closed circuit system of the experimental laboratory of the aforementioned Department of Hydraulics. In particular any rust or lose paint must be removed from the outside to provide a clean mounting position when installing the Doppler sensor. An adequately tared orifice meter was inserted (adequately 
downstream of the UDFM installation section) on this pipe that had a diameter $\mathrm{D}=0.100 \mathrm{~m}$, thanks to which it was possible to compare the flow rate values obtained using the UDFM. For a greater control, the test flows rates were also made to pass through a Thomson weir that was also accurately tared. In particular the precision tarring of the two measuring devices was carried out with a volumetric method. The comparison between the flow rates measured by the orifice meter and by the Thomson weir confirmed the reliability of the flow rate measured by the laboratory system. The comparison between the flow rate QD supplied by the UDFM and the flow rate QT from the laboratory measuring devices is reported in fig. 1.

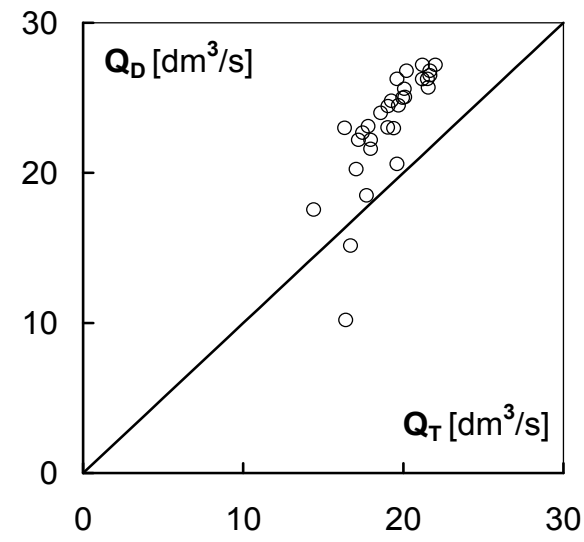

Figure 1: $\quad$ Experimental tests - series I.

Coinciding flow rate values for the various devices would of course imply that the experimental points be positioned on a straight line with passing through the origin at a $45^{\circ}$ angle (reference line). In fact in figure 1 it can be noted that: (i) for the greater flow rate values the UDFM gives strongly discordant results compared to the actual values; (ii) for intermediate flow rates corresponding to velocity values in the order of $2 \mathrm{~m} / \mathrm{s}$, the value provided by the UDFM tends to drop compared to the actual value; (iii) for lower flow rates corresponding to velocity values of less than $2 \mathrm{~m} / \mathrm{s}$ the UDFM does not give any result and the anomaly in its functioning is directly signalled by an LED that switches on in such cases. In fact the LED switches on precisely to indicate the system's inability to connect to a definite signal.

On the basis of such results and in order to investigate the reasons for this anomaly of the UDFM, a second series of tests (series II) was conducted under different experimental conditions. To this end the UDFM was mounted in the same way on the exterior of an acrylic pipe again with a diameter of $\mathrm{D}=0.100$ $\mathrm{m}$, connected to the closed circuit supply network of the laboratory. In this Series II in particular, the experimental tests concentrated on the range of the lowest flow rate values (corresponding to velocities inferior to $2 \mathrm{~m} / \mathrm{s}$ ) where the UDFM had not given any value. The results are reported in fig. 2, which has the same axis as the graph in fig. 1. 


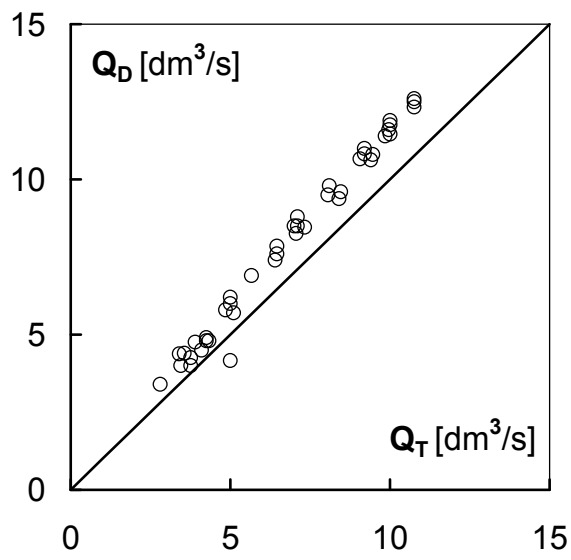

Figure 2: $\quad$ Experimental tests - series II.

The results obtained are very different: it can be noted that the UDFM worked up to extremely low flow rate values and indeed the fact that in fig. 2 no values inferior to the value of $0.003 \mathrm{~m}^{3} / \mathrm{s}$ is caused not by the UDFM, but by the impossibility of the laboratory measuring device to give acceptably precise values for such small flow rate values. In reality the UDFM continued to supply data (without the drop out LED switching on) up to flow rate values equal to about $0.0005 \mathrm{~m}^{3} / \mathrm{s}$, corresponding to a speed of about $0.05 \mathrm{~m}^{3} / \mathrm{s}$.

The experimental points (fig. 2) appear aligned along a straight line exiting from the origin, with an inclination slightly greater than $45^{\circ}$. This result testifies the linear response of the UDFM and simply highlights the necessity to correct the tarring constant that is normally applied to the operative procedure of this type of device.

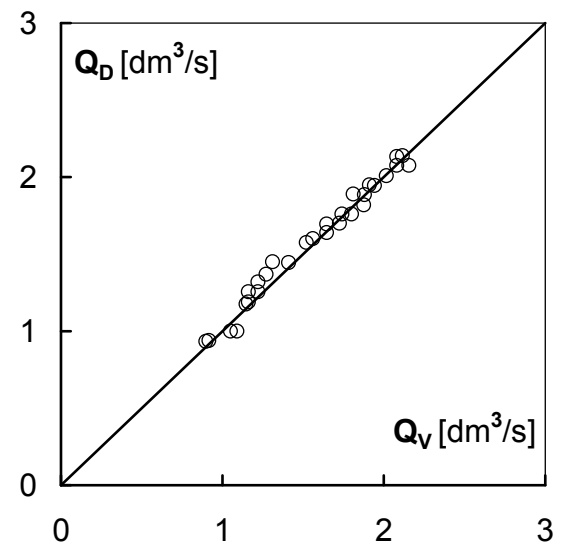

Figure 3: $\quad$ Experimental tests - series III. 
To complete the comparison in the smaller flow rate range, a third series of experiments (Series III) was carried out, having installed the UDFM on the exterior of a acrylic pipe with a diameter $\mathrm{D}=0.052 \mathrm{~m}$. The testing flow rates were made to pass through a small Venturi-meter that was also carefully tared. The results reported in fig. 3, in which the comparison between QD vs. QV can be deduced, substantially confirm the same considerations made in commenting the graph from fig. 2.

It must be underlined that, in Series III, a notable and greater adherence of the experimental points to the reference line illustrates the proper functioning of the UDFM in such experimental conditions.

\section{Discussion}

The two types of ultrasonic flowmeters described in the second section, though taking into account their different characteristics, present similar problems regarding their running and performance. Such circumstances have been highlighted both by users of this device and by authors of experimental work [5, 24-26]. In reality conditions occur whereby, apparently without explanation, the measuring device does not manage to supply reliable data or is unable to supply data at all, where under substantially identical conditions its functionality is flawless. The analysis of the experimental test results reported in the preceding section and obtained through an UDMF, in effect confirm this behaviour. In particular if the tests from Series I and Series II are considered, the only verifiable difference in the experimental conditions is inherent to the material the pipe is made of: steel for Series I and acrylic for Series II. Indeed the diameter of the pipe, the operating conditions, the measuring devices for the flow rate comparison, the quality of the fluid and, so, substantially the particle density concentration and distribution in the measure section, as well as the temperature and the sonic conductivity of the liquid are identical. Under these conditions the tests carried out on the steel pipe give strongly discordant values compared to the actual ones or do not give any data at all for the smaller flow rate values. On the contrary, despite the fact that many manufacturers of ultrasonic measuring devices tend to caution against the use of UFM on acrylic conduits (because they are wary of the possible presence of cracking internal to the material), the experimental tests conducted on the acrylic pipes highlight measurements of flow rate values close if not even coinciding with the actual values.

With the aim of deepening the accuracy of the UDFM, a further series of tests (Series IV) was carried out on the same acrylic pipe used in Series III. The experimental tests of Series IV were conducted on the range in which the device had shown the most measuring difficulty in the tests carried out on the steel pipe, i.e. the lower flow rate values $\left(1.2 \mathrm{dm}^{3} / \mathrm{s}-2.4 \mathrm{dm}^{3} / \mathrm{s}\right)$.

Firstly an error range was defined for the Venturi meter through which the measurement for the control of the flow rate is carried out. With this purpose a possible reading error of $1 \mathrm{~mm}$ was hypothesised on the level difference in the mercury column of the manometer connected to the Venturi meter. The error is equal to $20 \%$ in correspondence to the flow rate value relative to a level 
difference in the manometer of the Venturi meter of $0.05 \mathrm{~m}$ (corresponding to $1.2 \mathrm{dm}^{3} / \mathrm{s}$ ) and of $5 \%$ in correspondence to the flow rate value relative to a level difference of $0.20 \mathrm{~m}$ (corresponding to $2.4 \mathrm{dm}^{3} / \mathrm{s}$ ). This reading error spreads as a halved measuring error on the flow rate value (because of the formula containing a square root). Consequently we have an error of $10 \%$ (therefore of $0.12 \mathrm{dm}^{3} / \mathrm{s}$ ) in the case of the first flow rate value, and of $2.5 \%$ (therefore of $0.06 \mathrm{dm}^{3} / \mathrm{s}$ ) in the case of the second flow rate value. This evaluation permits in first approximation the estimate of the possible error range of the Venturi meter.

On the basis of experimental tests of Series III, a correction of the constant of the UDFM was carried out, determining its value with the method of minimum least squares (the correction that minimizes the difference between the experimental points and the reference line was sought). The value of the instrument constant was increased therefore by $3,4 \%$. Subsequently a series of tests was carried out (Series IV) the results of which are reported in figure 4, again accompanied by the reference line and by dotted lines defining the limit of the range of the possible error of the Venturi meter. The dotted lines that in fact should be curved, have been represented as strait for simplicity.

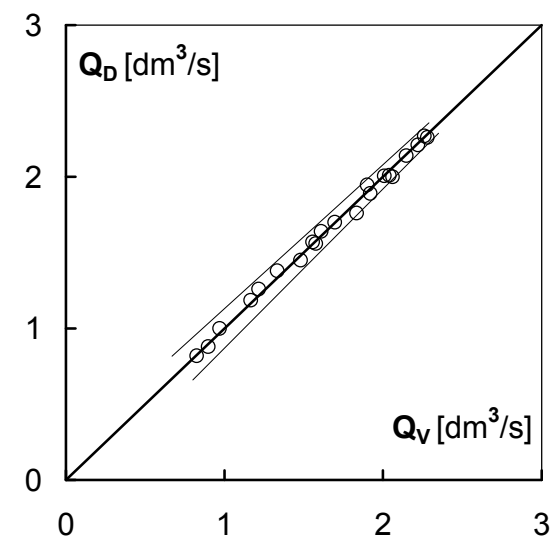

Figure 4: $\quad$ Experimental tests - series IV.

Fig. 4 shows that all the experimental points are positioned inside the range of error of the Venturi meter. This insures that errors of the UDFM can be considered negligible under the experimental conditions tested or, in any case, significantly inferior to those of the accurately tared Venturi meter using a volumetric method.

The remarkable accuracy obtained in the tests carried out on acrylic pipes prove that the anomalous functioning of the UDFM encountered in Series I should be attributed to the characteristics of the material constituting the steel pipe. Indeed the detailed analysis of the characteristics of the steel of which the pipe is composed led to the conviction that the anomalous behaviour of the measuring device is attributable to conformation of the internal surface of the pipe wall (moreover not immediately visible). In particular an alteration of the physical process in the acoustic signal passing through the internal surface 
of the pipe can be hypothesized. A very rough or even encrusted pipe wall can determine some undesired irregular refractions and a possible transmission loss or even a total black out of the signal, during the double passage of the ultrasonic beam through the uneven interface.

In other words a loss in the transmission coefficient occurs that is defined as the ratio between the power of the signal transmitted in the current and that of the signal emitted.

The tests relative to Series I, as previously stated, were conducted on one of the steel pipes constituting the closed circuit supply system of the experimental laboratory of the Department of Hydraulics of the University of Naples, which was built in the late sixties. The internal walls of such pipes thus present themselves as heavily rough and encrusted.

In conclusion it is possible to suppose that in the case of a steel pipe in such conditions the measuring device is unable to work up to a velocity in the pipe in the range of $2 \mathrm{~m} / \mathrm{s}$, while though giving velocity values for greater velocities it cannot guarantee the necessary precision. It must be emphasized that acrylic pipes that have extremely smooth internal surfaces, never presented any of the anomalies found in the old steel pipes in any of the tests conducted (Series II, III, IV).

\section{Conclusion}

Ultrasonic measuring systems have reached a level of significant reliability and of irreplaceable use in many fields of technology, of measuring and of NDT. In their use, however it is worthwhile to follow certain precautions. One of the specific problems that must be evaluated is connected precisely to one of the characteristics, which since the time of their appearance had made possible their major use, that is the non intrusiveness of the UFM.

The research carried out has shown that, beyond the worries of some producers on the use of UFMs on pipes made of plastic materials because of possible cracking inside the material, a much more limiting factor is the possibility of anomalous function of UFMs on working pipes in which significant roughness or incrustations may be present, which are of course not directly visible. Under such conditions the acoustic signal, in passing twice through the internal surface of the pipe, may lose its coherence and make it hard to pick up by the receiver.

When it is necessary to operate on installations composed of pipes that have been in use for long periods, the use of systems with clamp-on transducers mounted to the outside of the pipe wall, must be preceded by preliminary tests. In particular a first indication of efficiency can be obtained conducting preliminary tests in the range of small flow rate values in which (as has been deduced from experimental tests) the equipment tested had difficulty in acquiring data in the presence of considerable roughness. Furthermore it is worthwhile to conduct preliminary tests with the UDFM installed by drilling the pipe. Such tests on the whole constitute a sort of test of reliability and/or taring 
of the instruments that, on this basis, can be more opportunely used for any further measurements on the plant under observation.

Of course, the occurrence of anomalies like those encountered in the tests conducted in the present work would in any case imply the use of the UDFM installed via the drilling of the pipe, or through the fitting of a new section for transducer mounting. Naturally these last circumstances causes the measuring device to lose part of its quality of non intrusiveness and immediacy of use that make it extremely interesting, if not indispensable, in many fields both of technical practice and experimental research.

It must be stressed nonetheless that the experiments testify that the use of UDFMs on pipes presenting modest roughness show a very remarkable performance, corresponding to the best accuracy levels found in technical literature.

\section{References}

[1] Lynnworth, L.C., Waveguides in acoustic sensor systems, In: Chen $\mathrm{CH}$ editor. Ultrasonic and advanced methods for non-destructive testing and material characterization, Word Scientific Publishing Co, 10, pp. 217-244, 2007.

[2] American Society of Mechanical Engineers, Fluid meter their theory and application, Research Committee on Fluid Meter, Sixth edition revised New York NY: Bean HS editor, 1983.

[3] American National Standards Institute, Measurement of liquid flow in closed conduits using transit-time ultrasonic flowmeters, ANSI/ASME MFC-5M-1985, ASME United Engineering Center, 345 East 47th Street New York NY 10017, 1985.

[4] Furnes, R.A., Developments in pipeline instrumentation, Meas. and Contr., 20, pp. 25-38, 1987.

[5] Miller, R.W., Flow Measurement, Engineering Handbook, 3rd ed. USA, McGraw-Hill Co, 1996.

[6] Takeda, Y., Velocity profile measurement by ultrasound Doppler shift method, Int. J. Heat \& Fluid Flow, 7, p.313, 1986.

[7] Lynnworth, L.C., Ultrasonic Measurements for process control, New York: Academic Press Inc, 1989.

[8] Lohrmann, A., Cabrera, R. \& Kraus, N.C., Acoustic-Doppler Velocimeter (ADV) for Laboratory Use, Symposium Fundamentals and Advancements in Hydraulic Measurements and Experimentation, Buffalo, New York, ASCE, pp. 351-365, 1994.

[9] Vermeyen, T., Laboratory and Field Evaluation of Acoustic Velocity Meters, Symposium Fundamentals and Advancements in Hydraulic Measurements and Experimentation, Buffalo, New York, ASCE, pp. 43-52, 1994.

[10] Doering, J.C. \& Gawne, K.D., Developing a traversing acoustic discharge measurement technique for the performance testing of low-head hydroelectric turbines, Can. J. Civ. Eng., 25(4), pp.777-788, 1998. 
[11] Takeda, Y., Ultrasonic Doppler method for velocity profile measurement in fluid dynamics and fluid engineering, Exp. in Fluids, 26, pp. 177-178, 1999.

[12] Brown, A.E. \& Lynnworth, L.C., Ultrasonic flow-meters, In: Spitzer DW editor. Flow Measurements, USA: ISA, 20, pp. 515-573, 2001.

[13] Wang, T., Wang, J., Ren, F. \& Jin, Y., Application of Doppler ultrasound velocimetry in multiphase flow, Chem. Eng. J., 92, pp. 111-22, 2003.

[14] Laenen, A. \& Smith, W., Acoustic systems for the measurement of streamflow, US Geological Survey Water-Supply, Paper 2213, 1983.

[15] American Society for Testing and Materials, Open channel flow measurement by acoustic means, Standard Practice D 4408-84, 1916 Race Street, Philadelphia PA 19103, 1984.

[16] Sloat, J.V. \& Gain, W.S., Application of Acoustic Velocity Meters for gaging discharge of three low-velocity tidal streams in the St. Johns River Basin Northeast Florida, US Department of the Interior, US Geological Survey, Water Resources Investigation, Report 95-4230, 1995.

[17] Laplace, D. \& Deshons, P., Continuous flow rate measurements under a float. A new device for sewer network monitoring, 3rd Novatech Conference on Innovative Technologies in Urban Storm Drainage, Lyon (F), pp. 207-214, 1998.

[18] Larrarte, F., Velocity fields in sewers: an experimental study, Flow Meas. and Instr., 5, pp. 282-290, 2006.

[19] Muste, M., Vermeyen, T., Hotchkiss, R. \& Oberg, K., Acoustic velocimetry for riverine environments, J. Hydr. Eng., ASCE, 133(12), pp.1297-8, 2007.

[20] International Standard Organisation, Hydrometric determinations Liquid flow in open channels and partly filled pipes, Guidelines for the application of Doppler-based flow measurements, ISO/TS 15769, 2000.

[21] Rehmel, M., Application of acoustic Doppler velocimeters for streamflow measurements, J. Hydr. Eng., ASCE, 133(12), pp.1433-8, 2007.

[22] Larrarte, F., Bardiaux, J.B., Battaglia, P. \& Joannis, C., Acoustic Doppler flow-meters: A proposal to characterize their technical parameters, Flow Meas. and Instr, 19, pp. 261-267, 2008.

[23] US Department of the Interior Bureau of Reclamation, Water Measurement Manual, Water Resources Research Laboratory, Revised Reprint, Washington DC, 2001.

[24] Nolan, M.E., O'Hair, J.G. \& Teyssandier, R., The measurement of high pressure natural gas flows using the four-path ultrasonic flowmeter developed by British Gas, Int. Symposium on Flow Measurement, Washington DC, pp. 809-822, 1986.

[25] Calogirou, A., Boekhoven, J. \& Henkes, R.A.W.M., Effect of wall roughness on ultrasonic gas flowmeters, Flow Meas. and Instr, 12(3), pp. 219-229, 2001.

[26] Lynnworth, L.C. \& Liu, Y., Ultrasonic flowmeters: Half century progress report, 1955-2005, Ultrasonics, 44(1), pp. 1371-8, 2006. 